\title{
PROTOCOLO DE ATENÇÃO À SAÚDE DA MULHER COM HIV/AIDS: PERCEPÇÕES DE EQUIPE DE SAÚDE
}

\author{
HEALTHCARE PROTOCOL FOR WOMEN \\ WITH HIV/AIDS: PERCEPTIONS OF \\ THE HEALTH TEAM
}

\section{PROTOCOLO DE LOS CUIDADOS DE SALUD DE LAS MUJERES CON EL VIH/SIDA: LAS PERCEPCIONES DEL EQUIPO DE SALUD}

\author{
Jeniffer Stephanie Marques Hilário ${ }^{1}$ \\ Sabrina Pereira Lima ${ }^{2}$ \\ Jaqueline Silva Santos ${ }^{3}$ \\ Policardo Gonçalves Silva ${ }^{4}$ \\ Geilton Xavier de Matos 5 \\ Raquel Dully Andrade ${ }^{6}$
}

Como citar este artigo: Hilário JSM, Lima SP, Santos JS, Silva PG, Matos GX, Andrade RD. Protocolo de atenção à saúde da mulher com HIV/Aids: percepções de equipe de saúde. Rev baiana enferm. 2019;33:e33374.

Objetivo: descrever as percepções de uma equipe de saúde sobre o protocolo de atenção à saúde das mulheres vivendo com HIV implantado no serviço escola. Método: estudo descritivo com abordagem qualitativa, realizado com 10 membros de um serviço escola de referência regional para HIV/Aids. O grupo focal foi utilizado para a coleta de dados. Os depoimentos da(o)s participantes foram submetidos à análise de conteúdo, modalidade temática. Resultados: os temas construídos retrataram os impactos do protocolo na rotina do serviço de saúde e as contribuições da padronização para a atenção à saúde da mulher com HIV/Aids. A efetivação do protocolo foi compreendida como um processo contínuo que demanda envolvimento dos profissionais de saúde e reorganização de processos de trabalho. Conclusão: o protocolo implantado no serviço escola apareceu como uma ferramenta com potencial para facilitar o atendimento das mulheres cadastradas, com possíveis contribuições para a integralidade da assistência à mulher.

Descritores: Saúde da Mulher. Soropositividade para HIV. Protocolos. Qualidade da Assistência à Saúde. Integralidade em Saúde.

Objective: to describe the perceptions of a bealth care team on the healthcare protocol for women living with HIV deployed at the teaching service. Method: descriptive study, with a qualitative approach, carried out with 10

\footnotetext{
Enfermeira. Pesquisadora independente. Ribeirão Preto, São Paulo, Brasil. jsmhilario@outlook.com. https://orcid.org/0000-000 I-554I-6546

Estudante de Enfermagem. Universidade do Estado de Minas Gerais. Passos, Minas Gerais, Brasil. https://orcid.org/0000-0002-6559-8432

Enfermeira. Doutora em Ciências. Especialista em Políticas e Gestão da Saúde da Superintendência Regional de Saúde de Passos. Passos, Minas Gerais, Brasil. https:// orcid.org/0000-0002-9064-2490

4 Enfermeiro. Mestre em Ciências. Professor da Universidade do Estado de Minas Gerais. Passos, Minas Gerais, Brasil. https://orcid.org/0000-000 I-9095-6409

5 Farmacêutico. Mestre em Ciências. Professor da Universidade do Estado de Minas Gerais. Passos, Minas Gerais, Brasil. https://orcid.org/0000-000 I-7I72-7627

6 VEnfermeira. Doutora em Ciências. Professora da Universidade do Estado de Minas Gerais. Passos, Minas Gerais, Brasil. https://orcid.org/0000-0002- I5I 5-098X
} 
members from a regional teaching service of reference for HIV/Aids. The focus group was used for data collection. The participants' statements were submitted to content analysis, thematic modality. Results: the themes constructed portrayed the impacts of the protocol in the routine of the health service and the contributions of standardization for the health care of women with HIV/Aids. The execution of the protocol was understood as an ongoing process that requires the involvement of health professionals and reorganization of work processes. Conclusion: the protocol implemented in the teaching service appeared as a tool with the potential to facilitate the care with registered women, with possible contributions to the integrality of care provided to women.

Descriptors: Women's Health. HIV Seropositivity. Protocols. Quality of Health Care. Integrality in Health.

Objetivo: describir las percepciones de un equipo de atención de la salud sobre el protocolo de atención a la salud de las mujeres que viven con el VIH implementado en el servicio-escuela. Método: se realizó un estudio descriptivo con abordaje cualitativo, con 10 miembros de un servicio-escuela de referencia regional para VIH/SIDA. Se utilizó el grupo de enfoque para la recolección de datos. Los testimonios de los participantes fueron sometidos a análisis de contenido, modalidad temática. Resultados: los temas construidos retrataron el impacto del protocolo en la rutina de los servicios de salud y los aportes de la normalización de la atención de la salud de las mujeres con VIH/SIDA. La realización del protocolo fue entendida como un proceso continuo que requiere la participación de profesionales de la salud y la reorganización de los procesos de trabajo. Conclusión: el protocolo implementado en el servicio-escuela apareció como una berramienta con el potencial de facilitar el cuidado de las mujeres catastradas, con posibles contribuciones a la integralidad de la atención prestada a las mujeres.

Descriptores: Salud de la Mujer. Seropositividad para VIH. Protocolos. Calidad de la Atención de Salud. Integralidad en Salud.

\section{Introdução}

A atenção à saúde da mulher que vive com HIV/Aids apresenta-se como uma área delicada, que exige dos profissionais de saúde um olhar mais ampliado, voltado para as suas necessidades físicas, emocionais e sociais. Sabe-se que a epidemia de HIV/Aids não se limita a comportamento sexual, pois outros fatores, como gênero, condições de vida e composições etárias e étnicas, também se encontram envolvidos, o que aponta para a sua dinamicidade ${ }^{(1)}$.

As mulheres que vivem com HIV podem ser vistas pelos profissionais de saúde como vítimas ou culpadas ${ }^{(2)}$. Essa visão dicotômica indica que, na atenção à saúde, se torna premente a criação de espaços que possibilitem a fala das mulheres ${ }^{(3)}$ e a construção de laços de confiança, para evitar os preconceitos e os estigmas $^{(2)}$. Aqui, é importante pontuar que a estigmatização e as posturas discriminatórias aparecem como uma barreira que impossibilita, ao serviço de saúde, uma resposta condizente com as necessidades dessas mulheres ${ }^{(4)}$.

Tendo em vista que as intervenções dos serviços de saúde podem trazer benefícios para a mulher, assim como contribuir na adesão à terapia antirretroviral ${ }^{(3)}$, emergem as potencialidades de ferramentas voltadas para a construção de habilidades, para o seu empoderamento ${ }^{(5)}$ e ainda para a padronização da assistência ${ }^{(6)}$ a esse segmento.

Os protocolos, elementos técnicos embasados em evidências científicas ${ }^{(7)}$, configuram-se como ferramentas para a organização do serviço de saúde, que buscam a padronização de condutas assistenciais, a otimização do trabalho e a segurança do paciente ${ }^{(6)}$. A literatura aponta que os protocolos, na área de saúde, podem favorecer a gestão do cuidado $^{(8)}$. Por isso, é importante o levantamento e a descrição das percepções das equipes de saúde sobre essa temática. Nesse cenário, estudo realizado com integrantes da equipe de saúde de um serviço escola referência regional para HIV/Aids apontou percepções relacionadas ao entendimento de protocolos como instrumentos que podem resultar em maior segurança e qualidade no cuidado ao usuário ${ }^{(8)}$. 
O entendimento de que o HIV apresenta-se como algo crítico ${ }^{(3)}$, complexo e desafiador para a mulher, demanda um olhar mais atento dos profissionais de saúde ${ }^{(9)}$ às carências multidimensionais dessa mulher ${ }^{(10)}$, além de apontar para a necessidade de protocolos capazes de nortear as formas de cuidado em saúde ${ }^{(6)}$.

Diante da corresponsabilidade dos profissionais de saúde com a produção e o gerenciamento de um cuidado que atenda às premissas da integralidade e da humanização ${ }^{(11)}$, é fundamental pensar na percepção desses profissionais sobre a atenção integral à mulher com HIV.

O objetivo do presente estudo foi descrever as percepções de uma equipe de saúde sobre o protocolo de atenção à saúde das mulheres vivendo com HIV implantado no serviço escola.

\section{Método}

Trata-se de um estudo descritivo, com abordagem qualitativa, realizado em um serviço escola de referência regional para HIV/Aids, no interior do estado de Minas Gerais, Brasil. O estudo foi conduzido de acordo com os critérios expostos no Consolidated criteria for reporting qualitative research (COREQ) ${ }^{(12)}$.

O estudo descritivo possibilita a caracterização de determinado fenômeno ${ }^{(13)}$. A abordagem qualitativa busca a sistematização progressiva do conhecimento para o entendimento do fenômeno estudado, contribuindo para a construção de novas abordagens ${ }^{(14)}$.

No ano de 2018, foi elaborado e implantado um protocolo de atenção à saúde da mulher que vive com HIV no referido serviço. Esse protocolo continha os seguintes conteúdos: Saúde Mental da Mulher (alterações cognitivas, depressão, transtorno bipolar, uso de álcool e outras drogas em mulheres que vivem com HIV), Alterações Corporais na Mulher (lipodistrofia, alterações osteoarticulares relacionadas ao HIV), Mulher Soropositiva e a Sexualidade (cuidados com a mama, sintomas pré-menstruais, atraso menstrual, atraso menstrual - DUM $>12$ semanas -, atraso menstrual - descartado gestação -, aconselhamento para anticoncepção, climatério, queixas de lesão anogenital, cuidados com a vagina corrimento vaginal e cervicites - câncer de colo de útero -, Uso de Dolutegravir na Mulher que vive com HIV (mulheres em idade fértil que têm indicação de uso de Dolutegravir (dtg), mulheres em idade fértil em uso de Dolutegravir (dtg) e não usam contraceptivos eficazes, mulheres em idade fértil em uso de Dolutegravir (dtg) e fazem uso de contraceptivo definitivo), Gravidez e os Cuidados com a Mulher - gestantes HIV ou com suspeita de gravidez, gravidez, amamentação - e Rede de Apoio para as Mulheres.

Após um mês de implantação, foi realizada uma reunião, utilizando-se a técnica do grupo focal, com a equipe de saúde que atuava no serviço, composta por 10 membros, incluindo profissionais de saúde e acadêmicos estagiários bolsistas. Os critérios de inclusão dos membros foram: estar atuando no serviço, ser profissional ou acadêmico da área da saúde, ter contato direto com as mulheres cadastradas no serviço que vivem com HIV. O critério de exclusão foi não estar presente no serviço de saúde no dia e horário agendados para a reunião do grupo focal.

O grupo focal, utilizado na coleta de dados qualitativos $^{(15)}$, consiste em uma técnica voltada à produção de dados que possibilita espaços de problematização ${ }^{(16)}$, envolvimento e interação da(o)s participantes ${ }^{(15)}$. Além disso, pode funcionar como um facilitador para discussões e resolução de problemas, constituindo-se em uma oportunidade para a exploração de ações de saúde e estudos de implantação de programas ${ }^{(15)}$. No presente estudo, o intuito foi levantar as percepções da equipe sobre o processo de elaboração e implantação do protocolo de atenção à saúde das mulheres vivendo com HIV e identificar possíveis facilidades, dificuldades e sugestões em relação à efetivação desse instrumento no serviço de saúde escola.

A(o)s participantes do grupo foram 10 membros que compõem o serviço de saúde. Destes, 8 (80\%) eram do sexo feminino e 2 (20\%) do sexo masculino. A faixa etária variou de 20 a 50 anos: 4 (40\%), de 20 a 30 anos, e 6 (60\%), de 40 a 50 anos. Sobre a categoria profissional, 2 (20\%) são enfermeiros, 2 (20\%) são da área de 
nutrição (incluindo nutricionista e estagiário de nutrição), 1 (10\%) é técnico de enfermagem, 1 (10\%) farmacêutico, 1 (10\%) médico infectologista e 3 (30\%) eram estagiários do curso de enfermagem. Em relação ao tempo de atuação no serviço, 5 (50\%) atuavam há mais de cinco anos, 2 (20\%) trabalhavam há mais de dois anos, e 3 (30\%) estavam há aproximadamente um ano.

Para o desenvolvimento da técnica do grupo focal, é necessária a preparação e a instrumentalização prévia dos pesquisadores, bem como a flexibilidade e a criatividade no planejamento e na condução do encontro ${ }^{(16)}$, com ênfase no rigor metodológico ${ }^{(15)}$. Assim, inicialmente, a(o)s pesquisadoras realizaram leituras e reflexões sobre a técnica do grupo focal.

Tendo em vista a importância do processo de planejamento e organização para o desenvolvimento do grupo focal, foram seguidas as seguintes etapas: sensibilização dos profissionais participantes, preparação do ambiente, condução do encontro e análise dos dados coletados $^{(15)}$. Para a sensibilização, as pesquisadoras realizaram contato com os integrantes da equipe de saúde do serviço, convidando-os, face a face, para a participação no grupo focal. Não houve recusa.

A reunião do grupo focal ocorreu em uma sala do próprio serviço de saúde, em horário acordado para atender às necessidades dos integrantes da equipe de saúde. Nesse ambiente, as cadeiras foram dispostas em círculo, estratégia utilizada para promover a interação face a face e o contato visual entre a(o)s participantes ${ }^{(15)}$. O grupo focal contou com a participação de 12 pessoas (10 membros da equipe e duas pesquisadoras), e teve duração de aproximadamente 40 minutos.

É importante destacar os papéis desempenhados pelo moderador e pelo observador. O primeiro coordena as discussões do grupo focal, de forma harmoniosa e participativa; o observador exerce papel de apoio ${ }^{(15)}$. Para a condução do grupo focal, uma pesquisadora desempenhou o papel de moderadora, e a outra, o papel de observadora.
A moderadora acolheu a(o)s participantes, apresentou o Termo de Consentimento Livre e Esclarecido (TCLE), prestou esclarecimentos, explanou o conteúdo dos documentos governamentais que serviram de base para a formulação do protocolo, introduziu as questões norteadoras e conduziu o processo de discussão. A observadora exerceu o papel de apoio, auxiliando na organização da sala e no recolhimento dos TCLE assinados, por exemplo. Durante as discussões, a observadora também realizou anotações, em diário de campo, de aspectos importantes para a compreensão do objeto em estudo, observando a comunicação não verbal da(o)s participantes ao relatarem suas opiniões.

No grupo focal, as questões norteadoras são motivadoras do processo de discussão ${ }^{(15)}$. Por conseguinte, foi elaborado um roteiro composto pelas questões norteadoras utilizadas na condução do grupo: "Quais as facilidades de ter um protocolo de normas para o serviço?"; "Quais as dificuldades para adequar o protocolo à realidade do serviço?"; "O que achou do protocolo, em questão da sua estrutura, do conteúdo que abordou?"

Os depoimentos da(o)s participantes foram gravados em áudio, transcritos e analisados por meio da análise de conteúdo, modalidade temática, que busca a identificação de núcleos de sentido que compõem determinada comunicação, com significados para o indivíduo ${ }^{(14)}$. Destaca-se que não houve a apresentação dos dados transcritos à(ao)s participantes do estudo.

Neste artigo, a identificação da(o)s depoentes será feita pela letra $\mathrm{P}$ seguida do número de ordem da entrevista.

O projeto de pesquisa foi aprovado pelo Comitê de Ética em Pesquisa (CEP) da Universidade do Estado de Minas Gerais (UEMG), Parecer n. 2.333.413.

\section{Resultados}

Após a análise do conteúdo das falas da(o)s participantes, emergiram dois temas: a padronização da atenção à saúde da mulher: impactos 
do protocolo na rotina do serviço, e as contribuições da padronização para a atenção à saúde da mulher com HIV/Aids:

\section{Padronização da atenção à saúde da mulher: impactos do protocolo na rotina do serviço}

Os relatos da(o)s participantes do estudo apontaram para as potencialidades do protocolo, buscando melhorias na atenção à mulher com HIV. Para isso, emergiu a compreensão da necessidade de reestruturação de determinados processos de trabalho da equipe de saúde.

No relato a seguir, é enfatizada a importância da elaboração de protocolo condizente com a realidade e as necessidades do serviço:

\begin{abstract}
O protocolo foi elaborado de acordo com a nossa realidade, atendendo às necessidades do serviço na área da saúde da mulher. Só tenho a agradecer pela execução, pois os projetos de pesquisa que são realizados aqui auxiliam muito no gerenciamento do serviço, resultando em aprendizado para vocês [acadêmicos] e em contribuição valiosa para nós [profissionais]. (P1).
\end{abstract}

A utilidade do protocolo para o atendimento prestado por todos os integrantes da equipe, profissionais e acadêmicos foi enfatizada nos relatos a seguir:

Pessoalmente, achei o protocolo ótimo. Nós estamos trabalhando com ele no dia a dia do serviço. o protocolo vai ser muito útil para os novos profissionais e acadêmicos que vierem trabalhar aqui, pois terão um protocolo estruturado e detalhado desta área. (P1).

O protocolo encaixa na realidade dos nossos atendimentos. Aqui a rotatividade de alunos é algo que vem crescendo muito, tanto dos bolsistas quanto dos voluntários, e ele [o protocolo] vai ficar como base para o atendimento desta clientela, porque, para o servico funcionar, todos os profissionais precisam falar o mesmo conteúdo. Para os profissionais que estão há mais tempo, o conteúdo do protocolo é rotineiro, mas pra quem chega é muita informação. Além do mais que, cada caso precisa ser analisado de forma individual, para conseguirmos atingir a atenção integral. Então, para os profissionais que estão trabalhando há pouco tempo aqui ou para os futuros profissionais será uma referência para seguir, contribuindo para o envolvimento da equipe na área, tanto quanto os profissionais de longa data. (P2).

O reconhecimento da necessidade de continuidade do processo de implantação e utilização do protocolo pode ser vislumbrado nos relatos:
A criação do protocolo é muito válida. Será um legado que iremos seguir no serviço. Mesmo que a sua elaboração e implantação encerrar, vai continuar presente nos atendimentos das mulheres que vivem com HIV. (P4).

A equipe deve contribuirpara sua efetivação. Por mais que seja burocrático e tenha obstáculos na sua execução, vale a pena se esforçar um pouquinbo. (P7).

Não obstante o reconhecimento da importância do protocolo elaborado, foram relatadas algumas dificuldades para a efetivação dessa ferramenta, com destaque para o comparecimento da mulher ao serviço de saúde e para a criação de vínculo com a equipe:

A maior dificuldade que encontramos para efetivaro protocolo é a adesão da mulher no serviço, pois elas têm vergonha da situação que estão vivendo e querem ignorar o HIV. Uma das soluç̃es seria a criação de vínculos com as mulheres, a fim de obter todas as informações sobre a vida delas, demandando atenção mais especial da nossa equipe neste ponto. (P5).

A necessidade de reestruturação de alguns processos de trabalho da equipe também emergiu nos relatos:

Estamos executando uma reorganização da equipe para realizar o atendimento às mulheres. Está demandando tempo e muito trabalbo da equipe, mas está encaminhando. Já começamos a atender a clientela conforme o protocolo e está fluindo bem. (P10).

Uma das alternativas que propomos fazer, para facilitar a realização dos atendimentos integrais, é encaixar algumas consultas de mulberes do serviço, no mesmo dia, já que estarão aqui no serviço. (P8).

Com a padronização da assistência, o protocolo sistematiza o fluxo de atendimento para o serviço, no qual se define o papel de cada profissional na gestão do cuidado da mulher:

Facilitou para cada um de nós conbecermos melhor o trabalbo de cada um dos outros profissionais e a relação destes com o nosso próprio trabalbo, ampliando a compreensão sobre a continuidade da atenção à mulher. (P6)

Ficou mais claro para nós, com a implantação do protocolo, sobre o papel do enfermeiro na condução $e$ acompanhamento do caso, durante o fluxo de atendimento por cada profissional da equipe, e sobre a importância desta condução atenta, além do comprometimento de cada profissional envolvido, para que não haja lacunas no seguimento da mulber no serviço. (P3). 


\section{As contribuições da padronização para a atenção à saúde da mulber com HIV/Aids}

As percepções da equipe de saúde apontam para as contribuições advindas da padronização da assistência à saúde da mulher vivendo com o HIV/Aids. Observa-se o entendimento de que o olhar do profissional de saúde deve ser ampliado, voltando-se para as diferentes dimensões e necessidades da mulher:

\begin{abstract}
Estou vindo de um congresso agora, e uma das discussões mais prevalentes é a questão que a pessoa com HIV hoje vive bem mais, tem mais longevidade e, com isso, aumenta as doenças associadas ao HIV, e nós [os profissionais] teremos que aprender a conviver com essa situação. Então, o protocolo vem de acordo com as necessidades dessa população. (P1).
\end{abstract}

A mulher é muito vulnerável na sociedade, sendo importante um protocolo tão dedicado ao conbecimento das suas particularidades. (P7).

Assim, a(o)s participantes consideraram as potencialidades do protocolo para contribuições voltadas à integralidade do atendimento à saúde da mulher que vive com HIV/Aids:

O protocolo visa integralmente à mulher, colaborando para conbecê-la melhor, além do HIV, dando mais autonomia para os profissionais do serviço intervirem. (P3).

O protocolo vai auxiliar nos exames clínicos, as alterações que ocorrerem na mulher. E, por meio disso, avaliar a sua adesão ao tratamento e os efeitos da medicação, contribuindo para a melhoria da saúde dessas mulheres. (P6).

Além disso, a(o)s participantes reconheceram as contribuições de um atendimento integral para a resolutividade da atenção prestada pelo serviço, com repercussões positivas na saúde das mulheres:

Uma das vantagens é proporcionar à mulher uma atenção integral e, assim, melhorar sua saúde, para continuar enfrentando o HIV. (P5).

O protocolo tornou o serviço uniformizado e específico para as singularidades das mulberes e, assim, proporcionando maior resolutividade frente a diversos casos. (P9).

Diante da integralidade ofertada no atendimento, ocorreu humanização do profissional de saúde em relação à mulher, como evidencia $\mathrm{O}$ relato a seguir:

\begin{abstract}
A partir do protocolo, começamos a ver a mulher pelo todo, e isso demanda mais atenção por parte da gente [profissionais de saúde], gerando bumanização no cuidado à mulher, já que conbecemos cada usuária do serviço com suas especificidades e necessidades. Isso é ótimo! (P2).
\end{abstract}

A rede de apoio existente no serviço, sistematizada com a rede de atenção dos municípios, contribui para a qualidade da assistência final prestada às usuárias, segundo relatos a seguir:

\begin{abstract}
Outra coisa que já existia, mas que ficou sistematizado com a implantação do protocolo foi a relação do cuidado que é prestado no serviço com os outros serviços da rede de atenção dos municípios de abrangência, como, por exemplo, com as maternidades, as unidades de saúde da família, unidades de urgência e emergência e o Centro de Referência e Atendimento à Mulher em situação de Violência de Passos. (P10).

Melhorou a nossa comunicação com esses serviços $e$ as estratégias de referência e contrarreferência. O que acaba impactando na qualidade da assistência final prestada à mulher que vive com HIV. (P4).
\end{abstract}

\section{Discussão}

As(os) participantes deste estudo relataram facilidades e dificuldades encontradas na padronização da atenção à mulher com HIV em um serviço de saúde. O reconhecimento das potencialidades da ferramenta elaborada para a organização de processos de trabalho aponta para o entendimento que os protocolos configuram-se como instrumentos dinâmicos do processo de planejamento $^{(11)}$ nos serviços de atenção à mulher com HIV.

Assim, deve-se reconhecer as potencialidades no uso de protocolos pelos profissionais de saúde ${ }^{(11)}$. No presente estudo, foi observado que o processo de implantação do protocolo de atenção à saúde das mulheres vivendo com HIV destacou a necessidade de reorganização do serviço de saúde para o atendimento à mulher, de acordo com suas particularidades. Dessa forma, compreende-se que os profissionais de saúde devem identificar as reais necessidades de saúde da mulher ${ }^{(17)}$, para a organização de um cuidado condizente com a realidade vivenciada e com as demandas apresentadas. 
A organização de um cuidado desvinculado das necessidades da mulher pode contribuir para a ocorrência de lacunas na atenção às mulheres com HIV, como aquelas relacionadas à saúde sexual e reprodutiva ${ }^{(18)}$. Nesse cenário, o desenvolvimento de ações de promoção da saúde, que buscam o empoderamento dessa mulher ${ }^{(19)}$, também deve ser fomentado. Por conseguinte, a atenção à mulher com HIV deve buscar a promoção da saúde e a autonomia ${ }^{(18)}$.

Nos relatos da(o)s participantes pôde-se perceber que a padronização da assistência possibilitou contribuições para o atendimento integral, com possíveis repercussões na resolutividade da atenção à saúde da mulher vivendo com HIV/Aids. Há de se reconhecer que a integralidade pode ser traduzida no acesso a ações resolutivas, condizentes com as necessidades de saúde, que valorizam a multidimensionalidade da mulher ${ }^{(19)}$. Autores $^{(20)}$ reforçam que as pessoas com HIV/Aids têm direito a uma assistência integral, voltada para a garantia de acesso, direitos humanos e atendimento às reais necessidades de saúde.

Em relação à criação de vínculos entre a mulher vivendo com HIV e o profissional de saúde, há necessidade de estabelecimento de confiança. Apreende-se que a criação de vínculos mostra-se importante no cenário da integralidade do cuidado $^{(19)}$, pois possibilita uma aproximação com a realidade vivenciada por essa mulher e as necessidades de cuidado que ela apresenta.

Os serviços de atenção à mulher com HIV devem oferecer uma assistência de qualidade ${ }^{(21)}$. Tendo em vista que este estudo refere-se à realidade de um serviço de referência para HIV/Aids, acredita-se que a interlocução com os demais pontos da rede de atenção à mulher, com destaque para os serviços de Atenção Primária à Saúde (APS), possa contribuir para a garantia da integralidade do cuidado. Nessa direção, torna-se necessário problematizar o papel desempenhado por esses serviços no cuidado da mulher com HIV, importantes parceiros para o acompanhamento dessa mulher.

$\mathrm{Na}$ organização dos serviços de APS, as necessidades e as expectativas da população devem ser consideradas, buscando-se o acesso e a qualidade da atenção ${ }^{(20)}$. Dessa forma, é importante reforçar que, no cotidiano dos serviços de saúde, os protocolos, como ferramentas de gestão do cuidado, podem apresentar potencialidades que contribuem para a organização dos processos de trabalho e respaldam as ações dos profissionais de saúde $^{(8)}$. Todavia, estudo ${ }^{(8)}$ destaca que lacunas no envolvimento da equipe de saúde podem fragilizar o processo de padronização da assistência no serviço.

A APS aparece, portanto, como espaço promotor de saúde da população adscrita ${ }^{(21)}$, podendo assumir papel de destaque no desenvolvimento de ações voltadas aos usuários com $\mathrm{HIV}^{(20)}$. Por conseguinte, o cuidado compartilhado entre os diferentes serviços, APS e atenção especializada da rede de atenção à saúde ${ }^{(21)}$, mostra-se como uma oportunidade de integralidade na assistência à mulher com HIV. Esta pode vivenciar diferentes situações de vulnerabilidade, o que revela a importância de uma escuta sensível e humanizada dos profissionais de saúde durante os momentos de assistência ${ }^{(18)}$. Os relatos da(o)s participantes deste estudo reforçam a necessidade de o cuidado direcionar-se para as diferentes necessidades de saúde da mulher, não se restringindo aos aspectos biológicos, o que aponta a importância da ampliação do olhar do profissional de saúde para os diferentes cenários de vida da mulher ${ }^{(19)}$.

Questões sociais relacionadas ao estigma, por exemplo, foi outro aspecto abordado pelas(os) participantes deste estudo. Tendo em vista que a vivência de medos concernentes ao estigma social ainda se apresenta como uma realidade para mulheres com $\mathrm{HIV}^{(22)}$, as ações dos profissionais de saúde devem ser direcionadas para a garantia de direitos. Sabe-se que as condutas e as posturas adotadas pelos profissionais de saúde, como aquelas relacionadas ao respeito e à confidencialidade, podem refletir na continuidade do cuidado dirigido a essa mulher ${ }^{(22)}$.

Em um estudo ${ }^{(23)}$, as descobertas sugeriram que, além de atender às necessidades de saúde mental e ao estigma, é preciso desenvolver intervenções para reduzi-lo, orientando a comunidade e divulgando a prevenção de doenças 
entre mulheres vivendo com HIV. As intervenções de redução do estigma relacionado ao HIV visam mitigar seus efeitos internalizados. Uma das estratégias adotadas é a humanização no atendimento das mulheres pelos profissionais de saúde, criando vínculos ${ }^{(24)}$.

Ressalta-se que as mulheres precisam ser motivadas pelas equipes de saúde a conversar e discutir abertamente sobre sua situação, na tentativa de desestigmatizar a doença. Assim, precisam aprender a aumentar seu conhecimento sobre o problema de saúde, a tornar-se independentes em relação à sua saúde e a ter controle sobre suas decisões. Logo, as equipes de saúde devem fornecer apoio e cuidados de acordo com as necessidades delas ${ }^{(25)}$.

É importante reforçar que, muitas vezes, as posturas dos profissionais de saúde podem estar imbricadas por julgamentos, o que aponta a necessidade de estratégias de educação permanente ${ }^{(2,25)}$ direcionadas para o enfrentamento do estigma, de modo a contribuir para a ampliação do acesso das mulheres com sorologia positiva para o HIV aos serviços de saúde ${ }^{(3)}$.

Além disso, questões relacionadas ao sigilo e à confidencialidade também devem ser consideradas ${ }^{(20)}$, com contribuições ao estabelecimento e ao estreitamento do vínculo entre a mulher com HIV e os profissionais integrantes das equipes de saúde. Estudo ${ }^{(22)}$ aponta que aspectos referentes à qualidade do cuidado recebido e à garantia da integralidade podem repercutir na continuidade do cuidado. Este aspecto pôde ser observado em um estudo de revisão da literatura ${ }^{(25)}$, no qual verificou-se que as mulheres que estavam inseridas em grupos de apoio diminuíram o isolamento social e os sentimentos de vergonha, aumentaram a rede de amigos, criaram relações de empatia mútua, melhoraram os comportamentos de autocuidado e diminuíram o risco de reexposição ao HIV.

Após a implantação do protocolo de atenção à saúde das mulheres vivendo com HIV, é preciso que ele seja constantemente avaliado e atualizado. Sabe-se que os processos de avaliação, no campo da saúde, emergem como mecanismos intrinsecamente atrelados ao planejamento e à tomada de decisões ${ }^{(21)}$, o que repercute na qualidade da atenção prestada, em especial quando se trata de mulheres com HIV.

Este estudo apontou as potencialidades da organização do atendimento à mulher com HIV, considerando-a como um sujeito singular e multidimensional. Destarte, acredita-se que o acolhimento da mulher e o reconhecimento dos determinantes sociais do processo saúde-doença devem permear as ações de cuidado ${ }^{(19)}$ nos diferentes serviços de saúde. O entendimento é que o modo de organização desses serviços não deve funcionar como barreira à garantia do direito à saúde ${ }^{(20)}$. Assim, estratégias devem ser construídas, buscando-se o fortalecimento do cuidado à mulher com HIV ${ }^{(21)}$.

Uma limitação do estudo foi o fato de a pesquisa não ter abordado a percepção das mulheres que vivem com HIV, cadastradas no serviço, sobre a sistematização da assistência ocorrida após a implantação do protocolo, sugerindo-se, assim, novo estudo que avance no conhecimento acerca do olhar dessas mulheres sobre a atenção recebida.

\section{Conclusão}

O protocolo de atenção à saúde da mulher com HIV foi compreendido como uma ferramenta com potencial para facilitar o atendimento das mulheres cadastradas no serviço de saúde escola. Sua efetivação foi entendida como um processo contínuo que demanda envolvimento dos profissionais de saúde e reorganização de processos de trabalho. As percepções de equipe de saúde também estiveram atreladas às potencialidades da padronização para uma assistência integral à mulher, apontando para possibilidades de melhorias na qualidade e resolutividade do serviço.

Nesse sentido, acredita-se que este estudo possa contribuir para a ampliação do olhar sobre as diferentes formas de organização do atendimento à mulher com HIV/Aids, enfatizar a importância da reorganização dos serviços de saúde baseados em documentos governamentais e a necessidade de construção de um cuidado integral e longitudinal a essa mulher. 


\section{Colaborações:}

1 - concepção, projeto, análise e interpretação dos dados: Jeniffer Stephanie Marques Hilário, Sabrina Pereira Lima, Jaqueline Silva Santos, Policardo Gonçalves Silva, Geilton Xavier de Matos e Raquel Dully Andrade;

2 - redação do artigo e revisão crítica relevante do conteúdo intelectual: Jeniffer Stephanie Marques Hilário, Jaqueline Silva Santos e Raquel Dully Andrade;

3 - aprovação final da versão a ser publicada: Jeniffer Stephanie Marques Hilário, Sabrina Pereira Lima, Jaqueline Silva Santos, Policardo Gonçalves Silva, Geilton Xavier de Matos e Raquel Dully Andrade.

\section{Referências}

1. Santos NJS. Mulher e negra: dupla vulnerabilidade às DST/HIV/aids. Saúde soc. 2016;25(3):602-18.

2. Panarra BACS, Teixeira E, Palmeira IP, Rodrigues ILA, Ferreira AMR. Vítimas e culpadas: representações sociais sobre mulheres que vivem com HIV. Rev Cuid. 2017;8(3):1887-98.

3. Wechsberg WM, Ndirangu JW, Speizer IS, Zule WA, Gumula W, Peasant C. An implementation science protocol of the Women's Health CoOp in healthcare settings in Cape Town, South Africa: A stepped-wedge design. BMC Women's Health. 2017;17(1):85. DOI: 10.1186/ s12905-017-0433-8

4. Renesto HMF, Falbo AR, Souza E, Vasconcelos MG. Enfrentamento e percepção da mulher em relação à infecção pelo HIV. Rev Saúde Pública. 2014;48(1):36-42.

5. Wechsberg WM, Browne FA, Carney T, Myers B, Minnis A, MacDonald R, et al. The Young Women's Health CoOp in Cape Town, South Africa: Study protocol for a cluster-randomised trial for adolescent women at risk for HIV. BMC Public Health. 2018;18(1):859. DOI: 10.1186/ s12889-018-5665-5

6. Krauzer IM, Dall'Agnoll CM, Gelbcke FL, Lorenzini E, Ferraz L. A construção de protocolos assistenciais no trabalho em enfermagem. Reme - Rev Min Enferm. 2018;22:e-1087. DOI: $10.5935 / 1415-2762.20180017$
7. Azevedo CS, Miranda L, Sá MC, Grabois V, Matta G, Cunha M. Entre protocolos e sujeitos: qualidade do cuidado hospitalar em um serviço de hematologia. Cad Saúde Pública 2018;34(6):e00043817. DOI: 10.1590/0102-331X00043817

8. Pereira LR, Carvalho MF, Santos JS, Machado GAB, Maia MAC, Andrade RD. Avaliação de procedimentos operacionais padrão implantados em um serviço de saúde. Arq Ciênc Saúde. 2017:24(4):47-51.

9. Gomes JCR, Vieira BDG, Queiroz ABA, Alves VH, Rodrigues DP, Santos KM. A vivência de mulheres soropositivas para HIV/AIDS sobre o climatério: usuárias de um serviço especializado. Rev enferm UFPE on line. 2017;11(7):2765-72. DOI: 10.5205/ reuol.10939-97553-1

10. Teixeira M, Mendes MT, Borba KP, Pereira EM, Borba E. Avaliação da qualidade de vida em mulheres vivendo com HIV. Rev Enferm UFSM. 2015;5(2):360-7.

11. Vieira LJES, Silva ACF, Moreira GAR, Cavalcanti LF, Silva RM. Protocolos na atenção à saúde de mulheres em situação de violência sexual sob a ótica de profissionais de saúde. Ciênc saúde coletiva. 2016;21(12):3957-65.

12. Tong A, Sainsbury P, Craig J. Consolidated criteria for reporting qualitative research (COREQ): a 32-item checklist for interviews and focus groups. Int J Qual Health Care. 2007;19(6):349-57.

13. Gil AC. Como elaborar projetos de pesquisa. $5 \mathrm{a}$ ed. São Paulo: Atlas; 2010.

14. Minayo MCS. O desafio do conhecimento: pesquisa qualitativa em saúde. 12a ed. São Paulo: Hucitec; 2010.

15. Soares MI, Camelo SHH, Resck ZMR. A técnica de grupo focal na coleta de dados qualitativos: relato de experiência. Rev Min Enferm. 2016;20:e942. DOI: $10.5935 / 1415-2762.20160012$

16. Prates LA, Ceccon FG, Alves CN, Wilhelm LA, Demori CC, Silva SC, et al. A utilização da técnica de grupo focal: um estudo com mulheres quilombolas. Cad Saúde Pública. 2015;31(12):2483-92.

17. Albuquerque Netto L, Pereira ER, Tavares JMAB, Ferreira DC, Broca PV. Atuação da enfermagem na conservação da saúde de mulheres em situação de violência. Reme - Rev Min Enferm. 2018;22:e-1149. DOI: $10.5935 / 1415-2762.20160012$ 
18. Bellotto PCB, Lopez LC, Piccinini CA, Gonçalves TR. Entre a mulher e a salvação do bebê: experiências de parto de mulheres com HIV. Interface (Botucatu). 2019;23:e180556. DOI: 10.1590/interface. 180556

19. Coelho EAC, Silva CTO, Oliveira JF, Almeida MS. Integralidade do cuidado à saúde da mulher: limites da prática profissional. Esc Anna Nery Rev Enferm. 2009;13(1):154-60.

20. Melo EA, Maksud I, Agostini R. Cuidado, HIV/Aids e atenção primária no Brasil: desafio para a atenção no Sistema Único de Saúde? Rev Panam Salud Publica. 2018;42:e151. DOI: 10.26633/ RPSP.2018.151

21. Kleinubing RE, Eslava DG, Padoin SMM, Paula CC. Avaliação da atenção à saúde de gestantes com HIV: comparação entre serviço primário e especializado. Esc Anna Nery 2019;23(2):e20180258. DOI: 10.1590/2177-9465-EAN-2018-0258

22. Nzaumvila DK, Mabuza LH. Why do women not return for CD 4 count results at Embhuleni Hospital,
Mpumalanga, South Africa? Curationis. 2015 Jun;38(1). DOI: 10.4102/curationis.v38i1.1266

23. Ojikutu BO, Pathak S, Srithanaviboonchai K, Limbada M, Friedman R, Li S, et al. Community Cultural Norms, Stigma and Disclosure to Sexual Partners among Women Living with HIV in Thailand, Brazil and Zambia (HPTN 063). PLoS One. 2016 May;11(5):e0153600. DOI: 10.1371/ journal.pone. 0153600

24. Darlington CK, Hutson SP. Understanding HIV-Related Stigma Among Women in the Southern United States: A Literature Review. AIDS and Behavior. 2017;21(1):12-26.

25. Paudel V, Baral KP. Women living with HIV/AIDS (WLHA), battling stigma, discrimination and denial and the role of support groups as a coping strategy: a review of literature. Reprod Health. 2015 Jun;12:53. DOI: 10.1186/s12978-015-0032-9

Recebido: 30 de agosto de 2019 Aprovado: 7 de novembro de 2019 Publicado: 3 de fevereiro de 2020

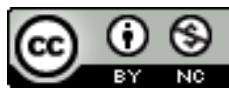

A Revista Baiana de Enfermagem utiliza a Licença Creative Commons - Atribuição-NãoComercial 4.0 Internacional. https://creativecommons.org/licenses/by-nc/4.0/ Este artigo é de acesso aberto distribuído sob os termos da Licença Creative Commons (CC BY-NC). Esta licença permite que outros remixem, adaptem e criem a partir do seu trabalho para fins não comerciais. Embora os novos trabalhos tenham de lhe atribuir o devido crédito e não possam ser usados para fins comerciais, os usuários não têm de licenciar esses trabalhos derivados sob os mesmos termos. 